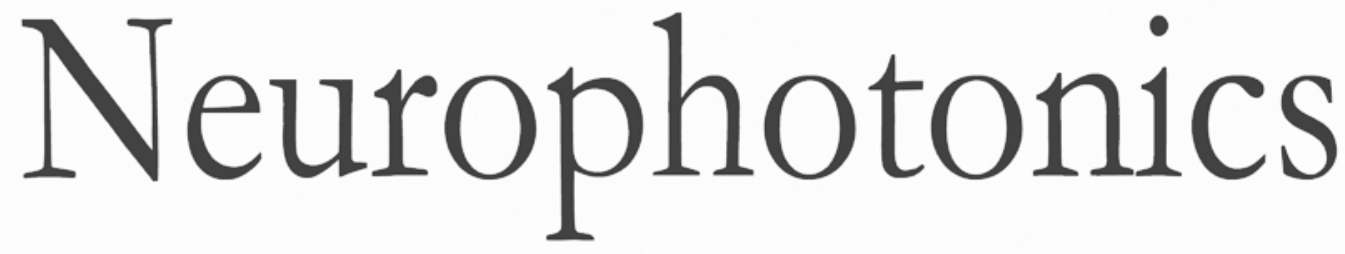

\title{
Multichannel fiber photometry for mapping axonal terminal activity in a restricted brain region in freely moving mice
}

\author{
Han Qin \\ Jian Lu \\ Wenjun Jin \\ Xiaowei Chen \\ Ling $\mathrm{Fu}$
}




\title{
Multichannel fiber photometry for mapping axonal terminal activity in a restricted brain region in freely moving mice
}

\author{
Han Qin, ${ }^{a, b}$ Jian Lu, ${ }^{c}$ Wenjun Jin, ${ }^{c}$ Xiaowei Chen, ${ }^{c}$ and Ling Fu ${ }^{a, b, *}$ \\ ${ }^{a}$ Huazhong University of Science and Technology, Britton Chance Center for Biomedical Photonics, Wuhan National Laboratory for \\ Optoelectronics, Wuhan, China \\ ${ }^{b}$ Huazhong University of Science and Technology, School of Engineering Sciences, Key Laboratory for Biomedical Photonics of Ministry of \\ Education, Wuhan, China \\ 'Third Military Medical University, Brain Research Center, State Key Laboratory of Trauma, Burns, and Combined Injury, Chongqing, China
}

\begin{abstract}
Fiber photometry has been increasingly popular in neuroscience research in freely behaving animals. In combination with genetically encoded calcium indicators, it allows for real-time monitoring of neural activity in neuronal somata, dendrites, and axonal terminals. We developed a multichannel fiber photometry device to map the activity of axonal terminals in a restricted, $100-\mu \mathrm{m}$-wide brain region in freely moving mice. This device consists of four bundled multimode fibers, each with a 50- $\mu \mathrm{m}$ core diameter and a scientific complementary metal-oxide semiconductor camera to simultaneously acquire fluorescence. We achieved a sampling rate of 100 frames/s and sufficient sensitivity to acquire data from axonal terminals. To avoid interference with neighboring channels, the recording depth of each channel was restricted to $<250 \mu \mathrm{m}$. Furthermore, the small-corediameter fibers did not restrict mouse locomotion. Using the $\mathrm{Ca}^{2+}$ indicator GCaMP5G, we validated the system by recording $\mathrm{Ca}^{2+}$ signals in axonal terminals from the medial entorhinal cortex layer II to the hippocampal dentate gyrus (DG) in freely moving mice. We detected spatially separated $\mathrm{Ca}^{2+}$ signals at four different sites in the DG. Therefore, our multichannel fiber photometry device provides a simple but powerful method to functionally map axonal terminals in spatially confined brain areas of freely moving animals. $\odot$ The Authors. Published by SPIE under a Creative Commons Attribution 4.0 Unported License. Distribution or reproduction of this work in whole or in part requires full attribution of the original publication, including its DOI. [DOI: 10.1117/1.NPh.6.3.035011]
\end{abstract}

Keywords: multichannel; fiber photometry; integrated probe; axonal terminals.

Paper 19032RR received Apr. 1, 2019; accepted for publication Aug. 5, 2019; published online Sep. 12, 2019.

\section{Introduction}

Axons transmit information from one neuron to another mainly through synapses at their terminals. Recording from axonal terminals in freely moving animals is an essential step to understand information processing during animal behaviors. A certain type of neuron in one area often projects to many different downstream regions with varying brain functions. ${ }^{1}$ The function and neural dynamics of the subdomains projected from the same cell population, with a distance of hundreds of micrometers, may also be different. For example, the retinal ganglion cells project to distant laminae zones of lateral geniculate nucleus and superior colliculus, which participate in different brain functions. ${ }^{2,3}$ Additionally, dopaminergic axons from the ventral tegmental area (VTA) project to both the nucleus accumbens core and shell, which play different roles in reward. ${ }^{4,5}$ Furthermore, the axonal terminals from the medial entorhinal cortex layer II (MECII) project to the entire region of the hippocampal dentate gyrus (DG), whose different subdomains may have different functions. Therefore, a method to record simultaneously from multiple sites in subdomains of an axonal projection target is greatly needed.

However, monitoring the activity of axonal terminals at multiple sites in freely behaving animals is challenging. Owing to their tiny sizes and high densities, recording the activity of axonal terminals using traditional electrophysiology is not

*Address all correspondence to Ling Fu, E-mail: Ifu@mail.hust.edu.cn feasible, especially in freely behaving animals. ${ }^{1,6,7}$ This situation changed when calcium $\left(\mathrm{Ca}^{2+}\right)$ imaging techniques, such as two-photon $\mathrm{Ca}^{2+}$ imaging, were developed, which enabled in vivo $\mathrm{Ca}^{2+}$ activity to be monitored at the micrometer scale. ${ }^{8,9}$ With the help of transgenetic or viral infection-based expression of genetically encoded $\mathrm{Ca}^{2+}$ indicators, ${ }^{10,11}$ twophoton imaging is particularly suited to record from superficial brain regions, like cerebral cortex, in anesthetized or head-fixed animals. ${ }^{12-14}$ Under these head-fixed conditions, two-photon imaging has been previously used to image neuronal activities at both axon buttons ${ }^{15,16}$ and dendritic spines. ${ }^{17,18}$ Two-photon imaging has also been adapted to record from multiple sites or large volumes; ${ }^{19,20}$ however, these applications are restricted to superficial brain regions of head-fixed animals as well. Recently developed miniaturized microscopes equipped with gradient refractive index (GRIN) lenses have been increasingly used in functional studies of deep brain regions in freely behaving animals. Dendrites and spines at significant tissue depths can even be recorded using a two-photon miniaturized microscope. ${ }^{21}$ However, this kind of technology has a restricted recording range in an individual brain region. Moreover, the use of the GRIN lens, with a typical diameter of $500 \mu \mathrm{m}$ or $1 \mathrm{~mm}$, causes larger invasion. ${ }^{22}$

Although it has poorer spatial resolution compared to the imaging approaches described above, fiber photometry can be used to record the activity of a group of neuronal cell bodies or axonal terminals in many brain regions in freely moving animals. ${ }^{1,7,23-31}$ Combined with genetic tools, one can perform 
fiber photometry recordings in axonal terminals in a cell-type and long-term manner. For example, Gunaydin et al. ${ }^{1}$ showed that the activity patterns of the axonal terminals from VTA to nucleus accumbens encoded and predicted key features of social behavior. We recently used this method to measure axonal terminal activity in the MECII-DG projection during place learning tasks. ${ }^{7}$ However, we were unable to determine whether the spatially distributed signals in the subdomains of the MECII-DG projection were homogenous or heterogeneous. To answer this question, we need to simultaneously record from the subdomains of the axonal terminals in freely behaving animals.

We previously developed a multichannel fiber photometry device that allowed us to record from three freely moving mice simultaneously. ${ }^{32}$ A recent study extended the recording channel up to seven channels and used it to record simultaneously from different brain regions receiving VTA axonal projections. ${ }^{33}$ Even with these advances, there is no multichannel fiber photometry device that can be used to simultaneously record from the subdomains of an axonal projection within a distance of only a few hundreds of micrometers. These studies used separated fiber ferrules and implanted each channel independently, which made the minimal distance of each neighboring channels more than $1.25 \mathrm{~mm}$. In addition, the fiber core diameter of these previous studies was $200 \mu \mathrm{m}^{32}$ or $400 \mu \mathrm{m},{ }^{33}$ which restricts the minimum distance without cross talk between neighboring channels. Furthermore, using optical fibers with larger diameters would cause greater mechanical stress and affect the locomotion of animals.

To enable simultaneous recordings from multiple axonal terminals in a restricted area such as the DG, we modified the fiber photometry device by adding a confined and integrated fiber probe with the neighboring channel close to $245 \mu \mathrm{m}$ to map axonal terminals. Equipped with a $50-\mu \mathrm{m}$ core diameter fiber, the recording depth was restricted to $<250 \mu \mathrm{m}$ and the mechanical constraint was reduced to a level that minimally affected mouse locomotion. In combination with a genetically encoded $\mathrm{Ca}^{2+}$ indicator, GCaMP5G, we then used this multichannel fiber photometry system to record neuronal activity from GCaMP5Gexpressing axons projecting from MECII to the DG in freely moving mice. We were able to record from four sites in the MECII-DG projection. We demonstrate a long-term and stable recording of the neuronal activity from the axonal terminals of this projection when the mice are freely exploring. Therefore, this work provides an effective approach to functionally map axonal terminals within a spatially confined brain region. In the future, the multichannel fiber photometry device can easily be extended to more than four channels.

\section{Methods}

\subsection{Multichannel Fiber Photometry Setup}

The framework of our custom-built multichannel fiber photometry system is shown in Fig. 1(a). Four individual multimode fibers were integrated into a bundle and then glued into a subminiature version A connector. The excitation light from a 488-nm semiconductor laser (Coherent, OBIS 488 LX, $50 \mathrm{~mW}$ ) was coupled into each fiber through an objective (Olympus, Plan N, 10×, NA 0.25 ) after being collimated with a beam expander [Fig. 1(a)]. The emitted fluorescence was separated from the excitation light through a dichroic mirror (Semrock, Di02-R488) and a bandpass emission filter (Semrock, FF01$535 / 22$ ). The images of the fiber end faces were captured by a scientific complementary metal-oxide semiconductor (sCMOS) camera (Hamamatsu, C11440-22C) with a sampling frequency of 100 frames/s [Figs. 1(b) and 1(c)]. The image of each fiber end-face was circled as a region of interest (ROI) and the averaged fluorescent intensity of each ROI was acquired as $\mathrm{Ca}^{2+}$ signal for each channel. The control of light excitation and the data acquisition were implemented by a custom-written software on the LabVIEW platform (National Instruments).

\subsection{Fabrication of Fiber Probe}

We utilized multimode $50-\mu \mathrm{m}$ diameter fibers (Thorlabs, FG050UGA, NA: 0.22 , core diameter: $50 \mu \mathrm{m}$, cladding diameter: $125 \mu \mathrm{m}$, and coating diameter: $245 \mu \mathrm{m}$ ), instead of the 200- $\mu \mathrm{m}$ diameter or $400-\mu \mathrm{m}$ diameter ones that have been used previously. ${ }^{32,33}$ Optical fibers were cut to create flat end faces and arranged into the shape of a square. Then a metal canula (0.62-mm inner diameter and 0.9-mm outer diameter) was used to bundle the fibers together [Fig. 2(a)]. To test the set-up, the laser beams from the fiber probe were illuminated onto a cardboard coated with fluorescent dye [Fig. 2(b)]. The power from each channel of the fiber probe was measured to confirm a uniform power distribution among the four channels [Fig. 2(c)]. The mean \pm standard deviation of power was $27.5 \pm 1.5 \mu \mathrm{W}$ with 3-mW illumination from laser.
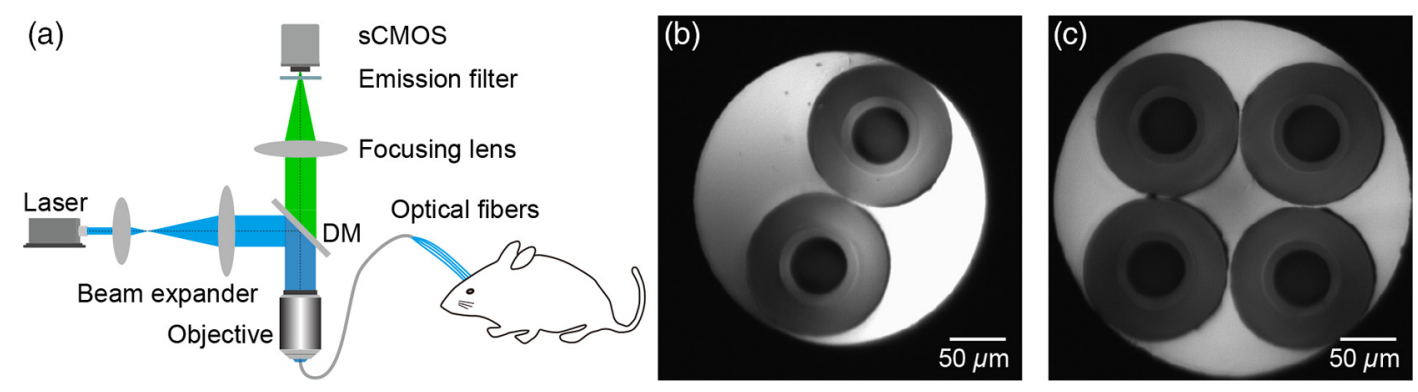

Fig. 1 The construction of multichannel fiber photometry system. (a) Overview of the multichannel fiber photometry. Light beam from a 488-nm laser is coupled into all multimode fibers simultaneously by an objective. Then the emitted fluorescence is collected by the same optical fibers and detected by a sCMOS camera. DM, dichroic mirror. (b), (c) Images of the fiber end faces on sCMOS camera for the dual-channel mode (b) and four-channel mode (c). 

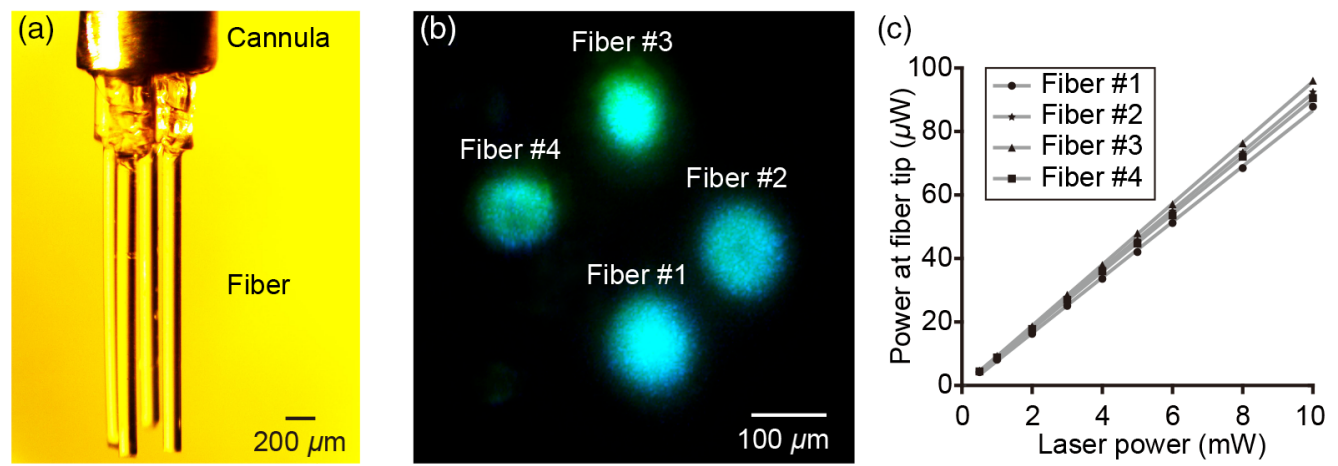

Fig. 2 The fabrication of multichannel fiber probe: (a) photograph showing the four-channel fiber probe which was arrayed into a form of square, (b) fluorescence image showing the excitation of fluorescent dye by multichannel fiber photometry, and (c) output power from the fiber probe as a function of laser power. Output power at the end of each fiber was measured showing a liner function of laser power and only slight difference among different channels.

\subsection{Animals}

C57/BL6J adult (2 to 5 months old) male mice were used in our experiments. Mice were housed in groups under a 12-h light/ dark cycle with free access to food and water. Mice were deprived of food $24 \mathrm{~h}$ before open field exploration. All procedures were performed in accordance with institutional animal welfare guidelines and were approved by the Third Military Medical University Animal Care and Use Committee.

\subsection{Virus Injection}

As described previously, ${ }^{7,34}$ mice were anesthetized with $1 \%$ to $2 \%$ isoflurane in pure oxygen and placed on a stereotactic frame. A warm pad was used to keep the animals at a proper body temperature $\left(36.5^{\circ} \mathrm{C}\right.$ to $\left.37.5^{\circ} \mathrm{C}\right)$. A small craniotomy with a diameter of $\sim 0.5 \mathrm{~mm}$ was made above $\mathrm{CA} 1$ ( $\mathrm{AP}=-2.0 \mathrm{~mm}$ and $\mathrm{ML}=$ $2.0 \mathrm{~mm})$ or $\mathrm{MEC}(\mathrm{AP}=-5.1 \mathrm{~mm}$ and $\mathrm{ML}=3.25 \mathrm{~mm})$. A glass micropipette with an inner tip diameter of 10 to $20 \mu \mathrm{m}$ was lowered down to the CA1 pyramidal layer or dorsal MEC to deliver the AAV2/9-syn-GCaMP5G virus. Approximately $100 \mathrm{~nL}$ of virus solution were injected into the brain tissue in $\sim 5 \mathrm{~min}$. The micropipette was held in place for $5 \mathrm{~min}$ before retraction. Tissue glue (3M Animal Care Products, Vetbond) was used for bonding the scalp incision.

\subsection{Histology and Fluorescence Imaging}

After neuronal activity recording, mice were transcardially perfused with $4 \%$ paraformaldehyde. Brains were then dehydrated with $15 \%$ sucrose in PBS solution before being cut into sagittal sections $(50 \mu \mathrm{m})$. Brain sections were then mounted on glass slides and covered by coverslips with mounting medium (Dako, C056330-2). Images were acquired with a wide-field fluorescence microscope (Olympus, BX43) to confirm virus expression and the position of the fibers.

\subsection{Data Analysis}

$\mathrm{Ca}^{2+}$ recording data were analyzed similar to previous work. $^{7,23,28}$ The data were filtered with a Savitzky-Golay FIR smoothing filter (five side points and three polynomial order). Then $\mathrm{Ca}^{2+}$ signals were calculated as

$\Delta f / f_{0}=\left(f-f_{0}\right) / f_{0}$, where $\left\{f_{0}\right\}$ is the average fluorescent intensity of baseline segments without transients. And then the signal-to-noise ratio (SNR) is: ${ }^{35}$

$\mathrm{SNR}=\left(\Delta f / f_{0}\right)_{\text {peak }} / \sigma f$,

where $\sigma f$ is the standard deviation of the baseline period. Events that exceeded an amplitude of 3 times the standard deviation of baseline were identified as $\mathrm{Ca}^{2+}$ events.

The correlation coefficient was calculated to evaluate the cross correlation of $\mathrm{Ca}^{2+}$ signals among different channels:

$r_{x y}=\frac{\sum_{i=1}^{n}\left(x_{i}-\bar{x}\right)\left(y_{i}-\bar{y}\right)}{\sqrt{\sum_{i=1}^{n}\left(x_{i}-\bar{x}\right)^{2}} \sqrt{\sum_{i=1}^{n}\left(y_{i}-\bar{y}\right)^{2}}}$,

where $x$ and $y$ represent the $\mathrm{Ca}^{2+}$ signals from different channels, $r_{x y}$ is the correlation coefficient, $\bar{x}$ and $\bar{y}$ are the mean values.

To evaluate asynchronized activity across channels, we calculated the proportions of local $\mathrm{Ca}^{2+}$ signals and global $\mathrm{Ca}^{2+}$ signals. $\mathrm{Ca}^{2+}$ events in two channels were paired in turn for comparison. $\mathrm{Ca}^{2+}$ events that were detected in a single channel were considered as local $\mathrm{Ca}^{2+}$ events, while ones detected in both channels were considered global $\mathrm{Ca}^{2+}$ events. The ratios of local $\mathrm{Ca}^{2+}$ events and global $\mathrm{Ca}^{2+}$ events to the total number of $\mathrm{Ca}^{2+}$ events were calculated.

Data were plotted as mean + SEM in figures. Nonparametric tests were used for data comparison, including Wilcoxon ranksum test for unpaired two-group test, Wilcoxon signed-rank test for paired two-group test, Kruskal-Wallis test for unpaired multigroup test and Friedman test for paired multigroup test. Results were considered significant if $p<0.05$.

\section{7 $\mathrm{Ca}^{2+}$ Event Detection}

We first assessed the $\mathrm{Ca}^{2+}$ event detection efficiency of our multichannel fiber photometry system using $50-\mu \mathrm{m}$ diameter fibers. Owing to weaker signals in axonal terminals than cell bodies, we compared the detection of signals recorded from axonal terminals with that from cell bodies by our system. ${ }^{1}$ To test this, we carried out simultaneous recording from cell bodies and axonal terminals in mouse CA1 pyramidal layer (Fig. 3). Adeno-associated virus (AAV) carrying GCaMP5G gene was injected into the CA1 pyramidal layer. One month 
(a) AAV-Syn-GCaMP5G

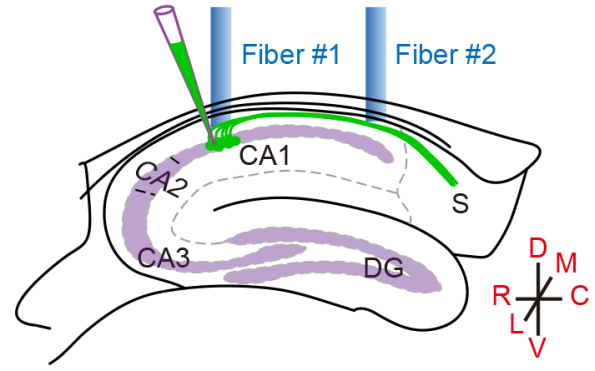

(b)

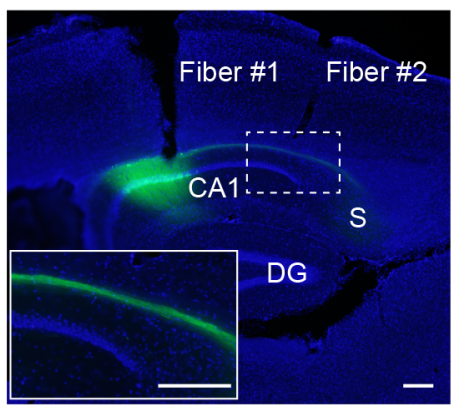

(c)

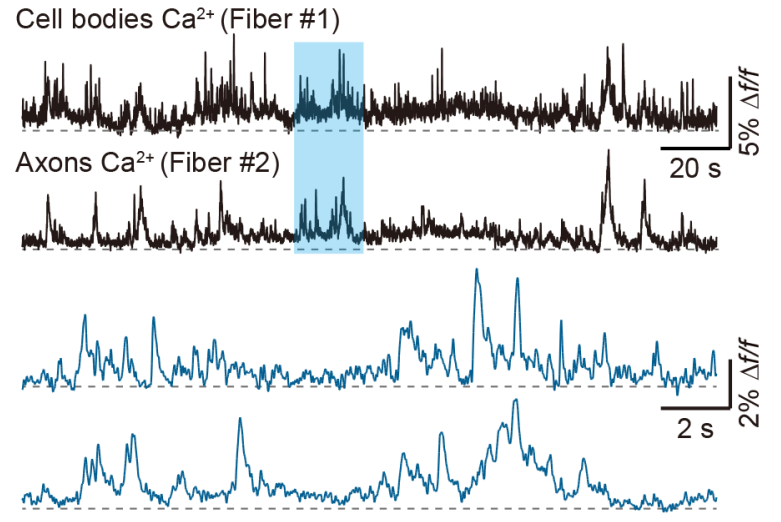

(d)

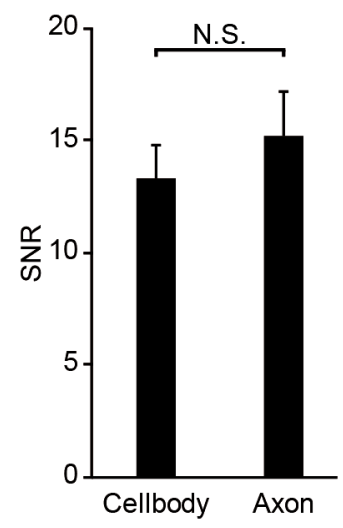

Fig. 3 The SNR of multichannel fiber photometry system in axonal terminals recording. (a) Diagram showing virus injection in $\mathrm{CA} 1$ pyramidal layer and $\mathrm{Ca}^{2+}$ recording at the virus injection site as well as the axonal terminals. R, rostral; C, caudal; D, dorsal; V, ventral; M, medial; and L, lateral. (b) Post hoc histological image showing GCaMP5G expression and the fiber tracks. Scale bars, $100 \mu \mathrm{m}$. Inset: the magnification of the dashed white box area. (c) Examples of $\mathrm{Ca}^{2+}$ signals recorded from cell bodies and axons in freely behaving mouse. Bottom panel showing the magnification of the light blue area in the upper panel. (d) Summary of the SNR of $\mathrm{Ca}^{2+}$ signals recorded from cell bodies and axonal terminals (Wilcoxon rank-sum test, $z=-0.27, p=0.79$, and $n=41$ trials).

after AAV-GCaMP5G injection, mice were anesthetized with $1 \%$ to $2 \%$ isoflurane in pure oxygen and placed on a stereotactic frame. Fiber \#1 was implanted above the virus injection site (AP: $-2.0 \mathrm{~mm}$, ML: $2.0 \mathrm{~mm}$, and DV: $1.2 \mathrm{~mm}$ ) to record $\mathrm{Ca}^{2+}$ signals from cell bodies and fiber \#2 was implanted $\sim 1 \mathrm{~mm}$ posterior to the injection site (AP: $-3.0 \mathrm{~mm}$, ML: $2.0 \mathrm{~mm}$, DV: $1.2 \mathrm{~mm}$ ) to record $\mathrm{Ca}^{2+}$ signals from axonal terminals [Fig. 3(a)]. The expression of virus and positions of fibers were confirmed by post hoc histology [Fig. 3(b)]. The $\mathrm{Ca}^{2+}$ signals from cell bodies and axonal terminals in freely behaving animals are shown in Fig. 3(c). The SNR of $\mathrm{Ca}^{2+}$ signals recorded from cell bodies and axonal terminals was not significantly different $(13.3 \pm 1.5$ for cell body and $15.3 \pm 2.0$ for axon, $p=0.79)$, suggesting that the sensitivity of our multichannel fiber photometry was sufficient for $\mathrm{Ca}^{2+}$ recording in axonal terminals [Fig. 3(d)].

\subsection{Recording under Different Laser Power}

Photobleaching occurs when using too much excitation power. Thus a suitable excitation power intensity should be chosen to balance the SNR and photobleaching. We recorded $\mathrm{Ca}^{2+}$ signals from axonal terminals under different excitation powers. Continuous recordings of $\mathrm{Ca}^{2+}$ activities from axonal terminals under different power densities at fiber tip (15.1, 10.0, 4.8, 2.2, and $15.1 \mathrm{~mW} / \mathrm{mm}^{2}$ in sequence) are shown in Fig. 4(a). SNR of $\mathrm{Ca}^{2+}$ activities increased with increasing excitation power densities [Fig. 4(b)]. Signal started to be distinguished from noise with a power density of $4.8 \mathrm{~mW} / \mathrm{mm}^{2}$ at fiber tip (with an amplitude larger than $3^{*} \mathrm{SD}$ of baseline and mean $\pm \mathrm{SEM}$ : $7.3 \pm 2.5$ ). Then we tested the photobleaching effect under a power density of $15.1 \mathrm{~mW} / \mathrm{mm}^{2}$. The recording was conducted continuously for 40 to $60 \mathrm{~min}$ [Fig. 4(c)]. The baseline decreased only $2.8 \%$ after a 40 -min continuous recording. The amplitudes and frequency of $\mathrm{Ca}^{2+}$ transients remained stable $\left[\chi^{2}=0.3, p=0.84\right.$; Fig. 4 (d) and $\chi^{2}=1.6, p=0.46$; Fig. 4(e)]. Therefore, a power density of $15.1 \mathrm{~mW} / \mathrm{mm}^{2}$ was chosen since it gave high SNR with minimal photobleaching.

\subsection{Recording Range of Single Channel}

We used $50-\mu \mathrm{m}$ core diameter fibers for recording to improve the mapping accuracy and reduce the recording range. We evaluated the recording range of our multichannel fiber photometry by theoretical calculation and experimental measurement.

We simplified the fiber probe from four channels into two channels for theoretical calculation. First light beams from two fibers propagated as shown in the schematic diagram in Fig. 5(a). The minimum distance of neighboring channels was $245 \mu \mathrm{m}$, the coating diameter of fiber. Therefore, we calculated the propagating distance from fiber tips before two beams overlapped using the function below: 
(a)
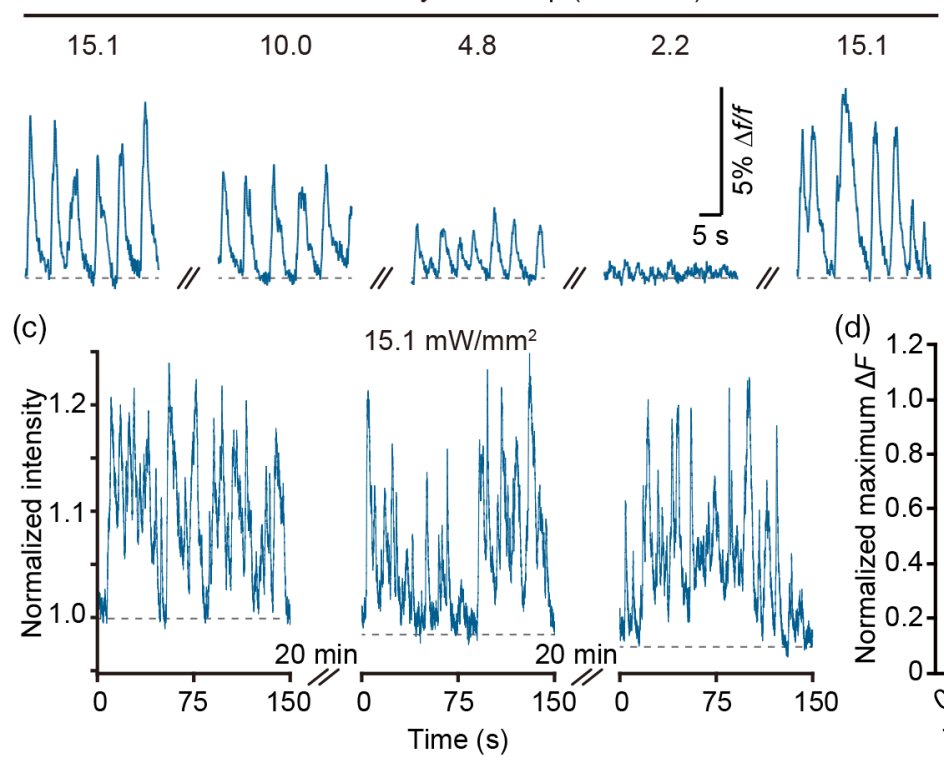
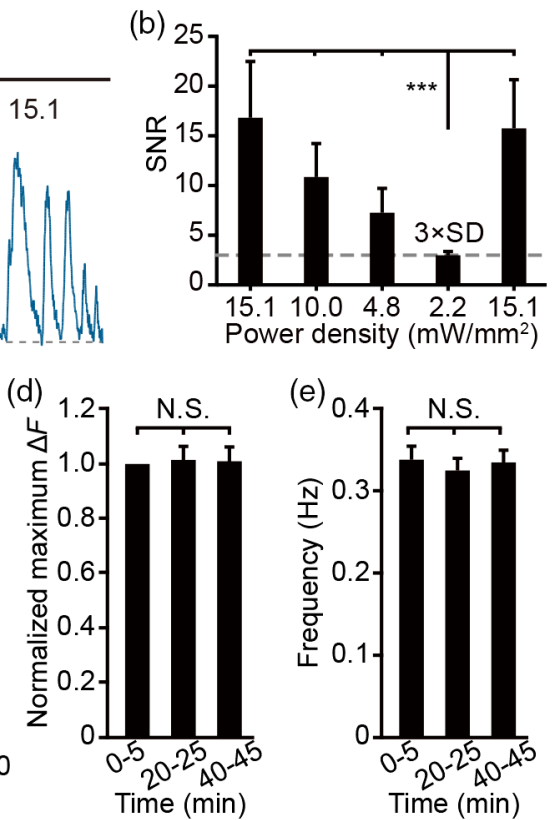

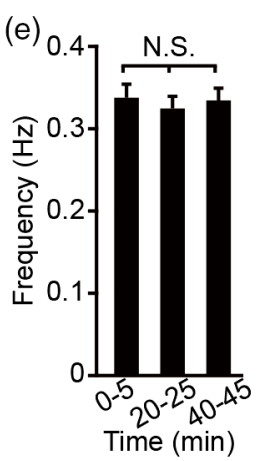

Fig. 4 The SNR and photobleaching effect of the multichannel fiber photometry system under different excitation powers. (a) Examples of $\mathrm{Ca}^{2+}$ signals under different excitation power intensities. (b) Summary of the SNR of $\mathrm{Ca}^{2+}$ signals under different power densities (Wilcoxon signed-rank test, $z=3.8$, ${ }^{* \star *} p<0.001$, and $n=20$ ). Gray dashed line, 3 times standard deviation of baseline. (c) The photobleaching effect of the multichannel fiber photometry. A continuous recording of more than 40 min was made under a power density of $15.1 \mathrm{~mW} / \mathrm{mm}^{2}$ at fiber tip. Three traces of $\mathrm{Ca}^{2+}$ signals with 20 min interval were plotted showing that the baseline decreased only $2.8 \%$ after a 40 -min recording. Data were normalized to the baseline of the first trace. (d) Summary of the maximum $\Delta F$ in the three recording periods. Data were normalized to the first trace. (Friedman test, $\chi^{2}=0.3, p=0.84$, and $n=20$ ). (e) Summary of the frequency of $\mathrm{Ca}^{2+}$ transients in the three recording periods. (Friedman test, $\chi^{2}=1.6, p=0.46$, and $n=20)$.

$$
S=\left(D / 2-r_{0} / 2\right) /\left\{\tan \left[\sin ^{-1}\left(\mathrm{NA}_{\text {fiber }} / n_{\mathrm{tis}}\right)\right]\right\}
$$

where $D$ was the distance of two fibers, $r_{0}$ was the core diameter of fiber, $\mathrm{NA}_{\text {fiber }}$ was the numerical aperture of fiber, and $n_{\text {tis }}=$ 1.36 was the refractive index of gray matter. ${ }^{36}$ The calculation results indicated that two light beams overlapped after a propagation of $600 \mu \mathrm{m}$.

Light intensity attenuates when propagated in tissue owing to light absorption and light scattering. Light scattering was mainly considered here because only minimal light absorption occurs in brain tissue at the wavelength range of 400 to $900 \mathrm{~nm} .{ }^{37}$ The light intensity after propagating in brain tissue could be simulated with the Kubelka-Munk model. ${ }^{36,38}$ The transmission coefficient $T$ is a function of the tissue depth $z$, as shown:

$T=\frac{1}{\mu_{s} z+1}$

where $\mu_{s}$ is the scatter coefficient, given as $11.2 \mathrm{~mm}^{-1}$ here. ${ }^{36}$ The power density at the depth $z$ is based on the energy conservation and geometry [Fig. 5(b)]:

$\frac{I_{z}}{I_{0}}=T\left(\frac{r_{0}}{r_{0}+z \tan \alpha}\right)^{2}$,

where $I_{z}$ and $I_{0}$ are the power density at the depth of $z$ and 0 from fiber tip, respectively, $r_{0}$ is the core diameter of fiber, $\alpha=\sin ^{-1}\left(\mathrm{NA}_{\mathrm{fiber}} / n_{\mathrm{tis}}\right)$ is the divergence angle of optical fiber. The attenuation curve was calculated and shown in Fig. 5(c).
The calculation predicted that light intensity would decrease to $\sim 0.5 \%$ of that at the tip after a propagation of overlapping distance $(600 \mu \mathrm{m})$.

Photons scatter in brain tissue which means that the fluorescence photon excited by one fiber may be scattered into the second fiber. Therefore, we conducted an in vivo experiment to directly measure the cross talk from neighboring channels. The experiment diagram is shown in Fig. 5(d). The fiber probe was slightly changed from previously described. Three channels were still connected to the multichannel fiber photometry system, whereas the last channel was individually attached to another $488 \mathrm{~nm}$ laser. The power at fiber tip was measured to guarantee a uniform light intensity. The fluorescent dye Oregon green 488 BAPTA-1 (OGB-1, $500 \mu \mathrm{M}$, O6807, Molecular Probes) was injected into C57 mouse cortex at the depth of $300 \mu \mathrm{m}(\sim 300 \mathrm{~nL})$. The fiber probe was implanted above the injection site. A normal recording where illumination was provided by the multichannel fiber photometry system was considered as control. The single-channel illumination group consisted of recording illumination only provided by the individual fiber. The images of three-fiber core end faces captured in the two illumination modes are shown in Fig. 5(e). The fluorescent intensities at three illumination power intensities are summarized in Fig. 5(f). The fluorescent intensity in the single-channel illumination group was $3.5 \% \pm 0.6 \%$ (mean \pm standard deviation) compared to the control, indicating that the cross talk among channels was negligible.

We also tried to find the recording depth by in vivo $\mathrm{Ca}^{2+}$ recording in the axonal terminals of GCaMP5G-expressing 


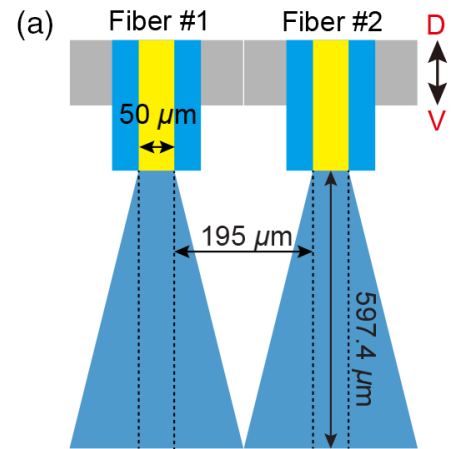

(d)

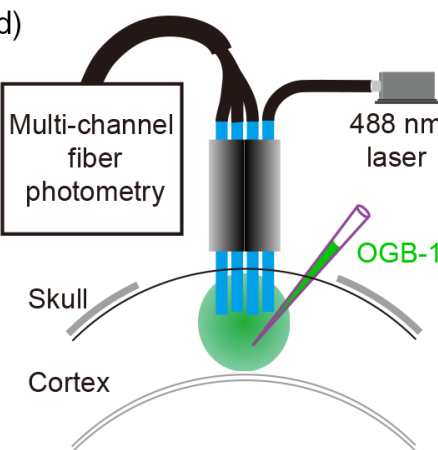

(b)

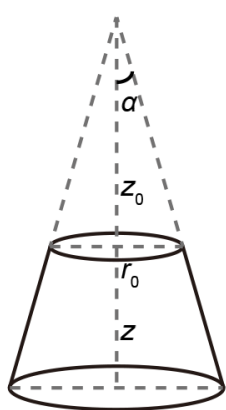

(e)

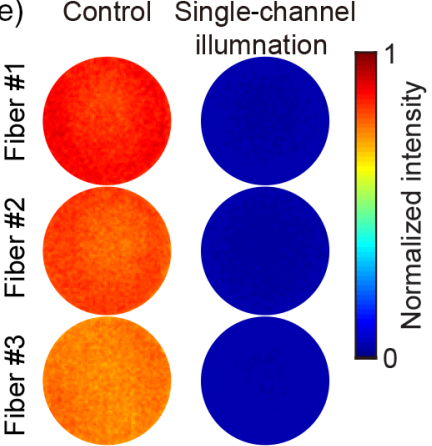

(c)

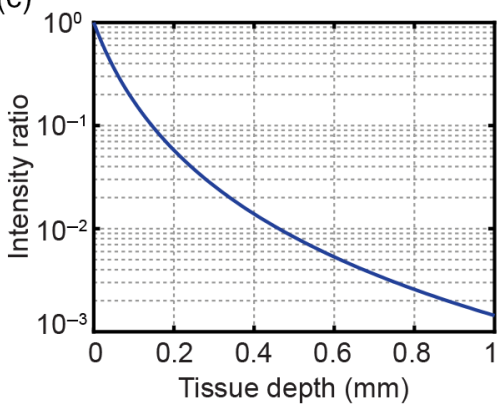

(f)

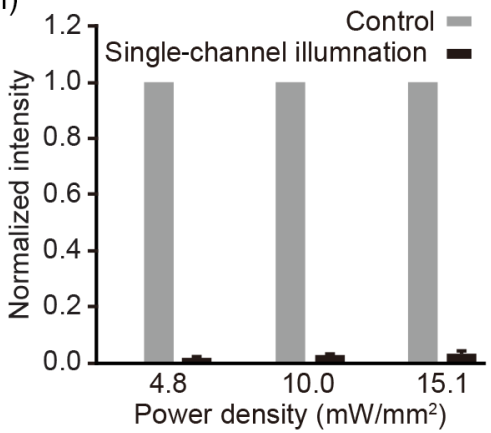

Fig. 5 Light propagating path from fiber tip and the cross talk from neighboring channel. (a) Diagram showing the propagating of laser beam from two neighboring fibers. (b) Geometric structure of laser beam after transmitted from fiber tip. (c) Attenuation curve of light intensity in different depths from tip $(50-\mu \mathrm{m}$ core diameter and NA $=0.22)$. (d) Diagram showing the fiber probe configuration for cross talk measurement. (e) Color map showing the fiber core images from three channels in the two illumination modes. Control group, illumination provided by multichannel fiber photometry system. Single-channel illumination group, illumination provided by an individual fiber. (f) Summary of the fluorescent intensity in the two illumination modes at three different illumination powers. Data were normalized to the control group.

mice. We carried out the recording as the fiber probe was advancing to the target in anaesthetized mice. The fiber probe was inserted into the brain tissue from the cranial window. The dura was carefully removed before implantation by fine forceps to confirm a smooth entry. Fiber probe was advanced with a speed of $50 \mu \mathrm{m}$ per min. At the depth of $1.2 \mathrm{~mm}$ from dura, we started to record the $\mathrm{Ca}^{2+}$ signal. After a 10-min continuous recording, fiber probe was advanced by a depth of $50 \mu \mathrm{m}$. The accurate distance to the target was measured with post hoc histology [Fig. 6(a)]. A power density of $\sim 15 \mathrm{~mW} / \mathrm{mm}^{2}$ at fiber tip was used. An example of recording at different depths was shown in Fig. 6(b), corresponding to the morphology in Fig. 6(a). The SNR of $\mathrm{Ca}^{2+}$ signals recorded at different depths is summarized [Fig. 6(c), $n=7$ mice]. The SNR increased as the fiber tip approached the target. $\mathrm{Ca}^{2+}$ signals could be distinguished from noise (with the amplitude larger than $3 \times \mathrm{SD}$ ) at 200 to $250 \mu \mathrm{m}$. Thus the $\mathrm{Ca}^{2+}$ recording depth was 200 to $250 \mu \mathrm{m}$ in our recording condition. For contrast, we also
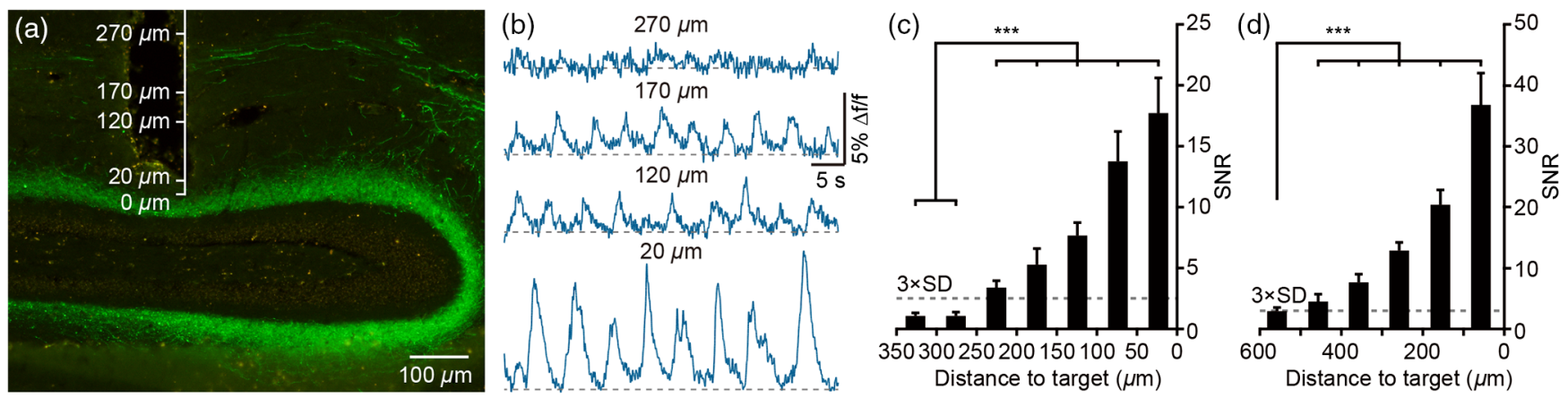

Fig. 6 The recording depth of multichannel fiber photometry system. (a) Post hoc histology after $\mathrm{Ca}^{2+}$ recording; (b) examples of $\mathrm{Ca}^{2+}$ signals in four different depths [shown in (a)] relative to the axonal terminals in anesthetized state. (c) Summary of SNR of $\mathrm{Ca}^{2+}$ signals recorded by multichannel fiber photometry system in different depths. Gray dashed line, 3 times standard deviation of baseline (KruskalWallis test, $\chi^{2}=84.5,{ }^{* \star \star} p<0.001$, and $n=28$ recording sites from 7 mice). (d) Summary of SNR of $\mathrm{Ca}^{2+}$ signals recorded by single-channel fiber photometry system in different depths (200- $\mu \mathrm{m}$ diameter, 0.48 NA fiber, Kruskal-Wallis test, $\chi^{2}=34.5,{ }^{\star \star \star} p<0.001$, and $n=7$ mice). 
measured the recording depth of the previously developed single-channel fiber photometry system [Fig. 6(d), $n=7$ mice]. We used a $200-\mu \mathrm{m}$ diameter and 0.48 NA multimode fiber for detection. The power density at fiber tip was about $0.22 \mathrm{~mW} /$ $\mathrm{mm}^{2}$. The recording depth was measured to be $\sim 400$ to $500 \mu \mathrm{m}$, which was similar to the results in previous studies. ${ }^{23,25}$ Then the recording volume of each single-channel can be calculated according to the volume of frustum of a cone [Fig. 5(b)]:

$$
\begin{aligned}
V= & \frac{1}{3} \pi\left[\left\{r_{0} / 2+z \tan \left[\sin ^{-1}\left(\mathrm{NA}_{\text {fiber }} / n_{\mathrm{tis}}\right)\right]\right\}^{2}\left(z+z_{0}\right)\right. \\
& \left.-r_{0}^{2} z_{0} / 4\right]
\end{aligned}
$$

where $z_{0}=r_{0} / 2 / \tan \left[\sin ^{-1}\left(\mathrm{NA}_{\text {fiber }} / n_{\text {tis }}\right)\right]$. Calculations showed that the single-channel recording volume of our multichannel fiber photometry system was 1.13 to $1.73 \times 10^{6} \mu \mathrm{m}^{3}$. Furthermore, the recording volumes of previously developed fiber photometry systems were 4.11 to $6.40 \times 10^{7} \mu \mathrm{m}^{3}$ for 200- $\mu$ m diameter, 0.48 NA fiber and 9.78 to $14.1 \times 10^{7} \mu \mathrm{m}^{3}$ for $400-\mu \mathrm{m}$ diameter, 0.48 NA fiber. Therefore, the recording volume of our multichannel fiber photometry system decreased to $\sim 1 \%$ to $3 \%$ of the previously developed ones.

\subsection{Mechanical Constraint}

The optical fibers could impose a mechanical constraint to freely moving mice, affecting their ability to freely access the entire recording environment. To test for this, we recorded from freely behaving mice exploring a $1.2-\mathrm{m}$ diameter round basin [Fig. 7(a)] with the $\mathrm{Ca}^{2+}$ signals and behavior video recorded simultaneously. The optical fibers we used were 4- to 5-m long, and the recording system was put at the north side of the basins [see the azimuth diagram in Fig. 7(e) inset]. Videos were captured at a frame rate of $50 \mathrm{~Hz}$ under infrared light illumination (Cannon, XA-25, $1920 \times 1080$ pixels). The location of the mouse was tracked using a custom MATLAB program [Fig. 7(b)] and 20-min recording of each mouse was used for analyses. Mice implanted with our multichannel fiber probe were formed multichannel group, whereas mice implanted with single-fiber patch cable $(200 \mu \mathrm{m}$ and $0.48 \mathrm{NA})$ were singlechannel group. First, the mean locomotion speed was compared between multichannel group and single-channel group, showing a lower speed in the multichannel group [multichannel group: $2.7 \pm 0.16 \mathrm{~cm} / \mathrm{s}, \quad n=9$ mice; single-channel group: $3.6 \pm$ $0.24 \mathrm{~cm} / \mathrm{s}, n=10$ mice, $z=2.8, p<0.01$, Fig. 7(c)]. To quantify the restriction by the optical fibers, we divided the basin into three regions with different diameters but the same area and named these: inner part, middle part, and outer part [Fig. 7(b)]. The time that mice stayed in these three regions is summarized in Fig. 7(d). We found no significant difference in the time spent in each of the three regions from multichannel group (black, $\chi^{2}=2.0$ and $p=0.37$ ). Additionally, mice in the singlechannel group also showed similar time distribution in the three divided parts (gray, $\chi^{2}=0.8$ and $p=0.67$ ). We then divided the basin space into four quadrants located in four directions [Fig. 7(e), inset]. There was no significant difference in the time spent in each of the four quadrants in the multichannel group $\left[\chi^{2}=4.1, p=0.25\right.$, Fig. 7(e) black bar $]$ nor the control single-channel group $\left[\chi^{2}=4.0, p=0.27\right.$, Fig. 7(e) gray bar]. This indicated that the fibers had no visible effect on the moving range of mouse.

\section{Results}

The MEC provides information to hippocampus from cerebral cortex and transmits processed messages back to cerebral cortex. ${ }^{39,40}$ The MECII-DG projection, the initial phase of the trisynaptic circuit (MECII-DG-CA3-CA1), transmits spatial information to DG. ${ }^{7,41,42}$ The neural representation at different subdomains of the MECII-DG projection during free exploration remains unknown. To address this question, we simultaneously recorded $\mathrm{Ca}^{2+}$ signals at multiple MECII-DG projection sites with our multichannel fiber photometry in freely exploring mice.

To monitor the $\mathrm{Ca}^{2+}$ activities in the MECII-DG projection, we first injected AAV-syn-GCaMP5G into MEC to label this pathway. A fiber probe with four channels was then implanted above the MECII-DG projection [Fig. 8(a)]. The expression of virus in the MECII-DG projection was confirmed by post hoc histology [Figs. 8(b) and 8(c)]. Most of the cells expressing GCaMP5G in the injection site were in MEC layer II [Fig. 8(b)]. The axonal terminals in DG were also labeled with GCaMP5G [Fig. 8(c)]. The configuration of the axonal terminals in DG from MECII was a U-shape in rostral-caudal direction [Fig. 8(c)]. We measured the width of the dorsal side of the MECII-DG
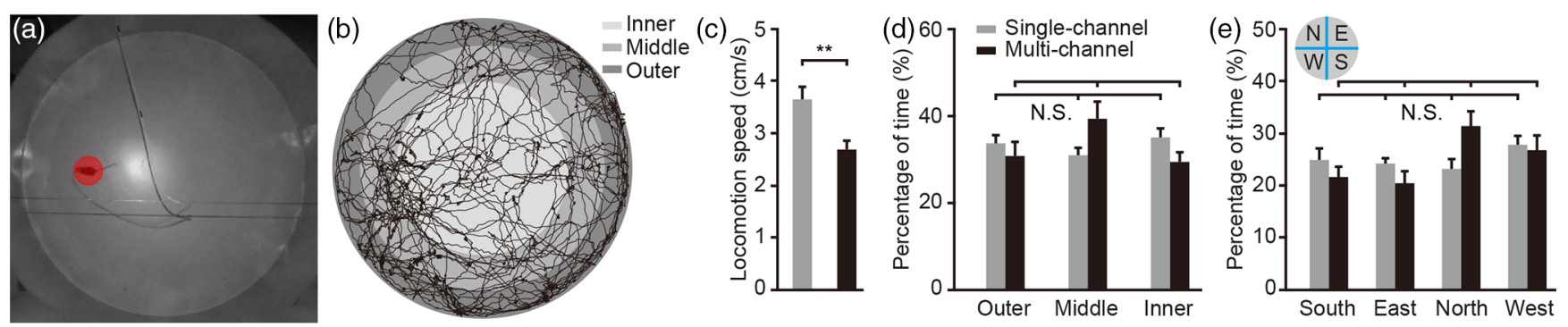

Fig. 7 Mechanical constraint imposed by fibers. (a) Recording scene in a large circular basin with a diameter of $1.2 \mathrm{~m}$. (b) Example of mouse trajectory after a freely exploring of $\sim 30 \mathrm{~min}$. The circular basin was divided into three parts with an equal area but different diameters named as inner part, middle part, and outer part. (c) Summary of the locomotion speed in multichannel and single-channel group. Black, multichannel group, $n=9$ mice; gray, single-channel group, $n=10$ mice (Wilcoxon rank-sum test, $z=2.8,{ }^{*} p<0.01$ ). (d) Summary of the time distribution in the three different parts of multichannel and single-channel group (Friedman test, multichannel group: $\chi^{2}=2.0, p=0.37$, and $n=9$ mice; singlechannel group: $\chi^{2}=0.8, p=0.67$, and $n=10$ mice). (e) Summary of the time distribution in the four quadrants of multichannel and single-channel fiber implantation group. Inset: diagram showing the quadrant division (Friedman test, multichannel group: $\chi^{2}=2.0, p=0.37$, and $n=9$ mice; single-channel group: $\chi^{2}=0.8, p=0.67$, and $n=10$ mice). 

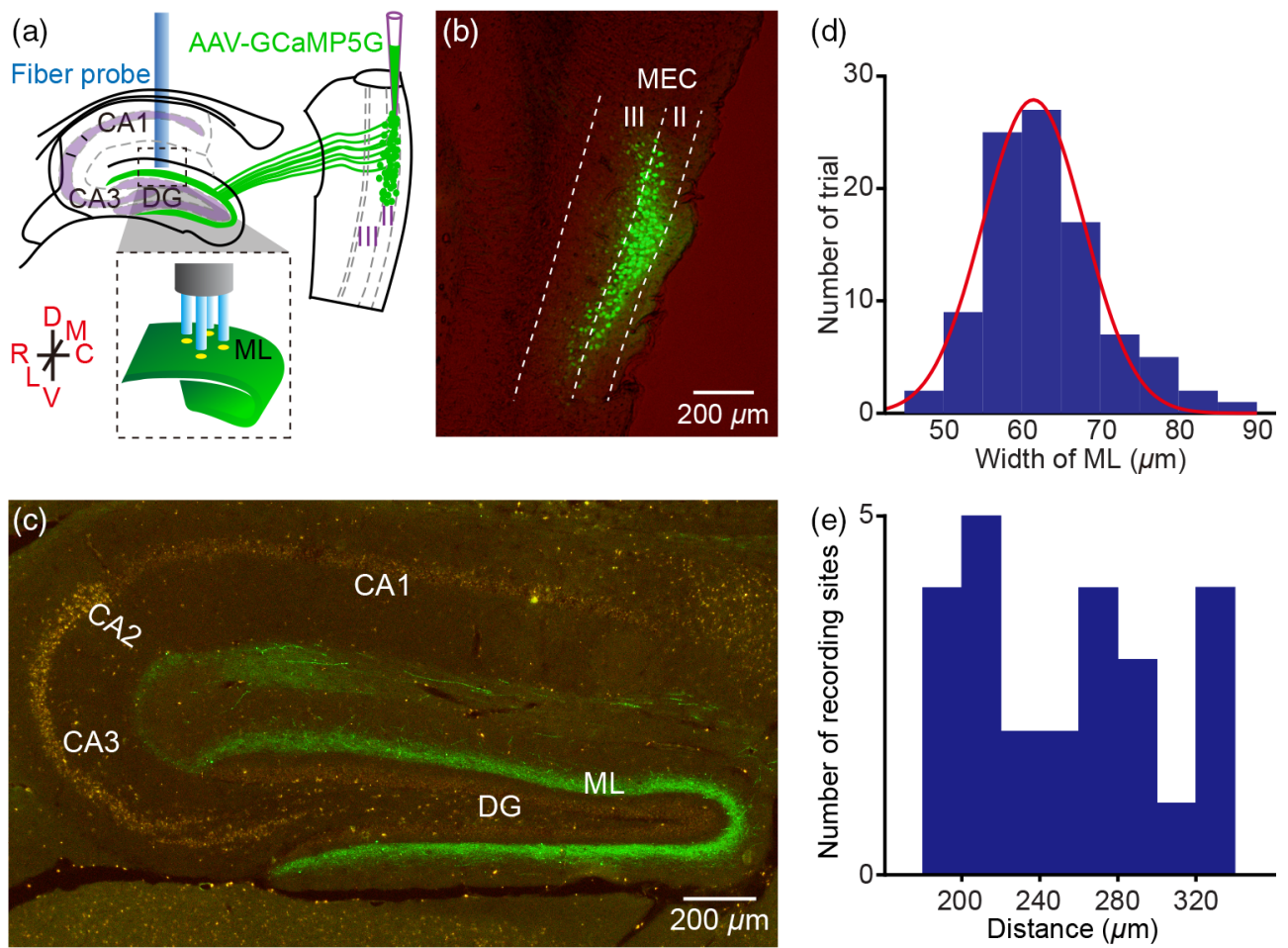

Fig. 8 MECII-DG pathway labeled by GCaMP5G through virus injection. (a) Diagram showing virus injection in MECII and multiple sites $\mathrm{Ca}^{2+}$ recording at the axonal terminals site of stratum molecular of DG (ML, stratum molecular of DG). (b), (c) Post hoc histology showing GCaMP5G expression in the injection site MEC (b) and axonal terminals in DG (c). (d) Distribution of the width of ML (Gaussian fit, mean $=64 \mu \mathrm{m}, R^{2}=0.96$, and $n=95$ from 10 mice). (e) Distribution of distance from the dorsal edge to ventral edge of $\mathrm{ML}$ ( $n=25$ from 7 mice).

projection (sagittal section), which was $64 \pm 2.4 \mu \mathrm{m}$ [Fig. 8(d), $n=10$ mice]. The distance between the dorsal and ventral side of the projection was $260 \pm 22.2 \mu \mathrm{m}$ [Fig. 8(e), $n=7$ mice]. Therefore, $\mathrm{Ca}^{2+}$ signals on the ventral side of the DG could not be detected if the fiber tip was above the dorsal side due to the recording depth of 200 to $250 \mu \mathrm{m}$.

One month after AAV-GCaMP5G injection, the prepared fiber probe was implanted through a cranial window to the DG (AP: $-2.0 \mathrm{~mm}$ and ML: $2.0 \mathrm{~mm}$ ) at a depth of $\sim 1.8 \mathrm{~mm}$ below the pia surface. The locations of the four individual channels were confirmed by post hoc histology [Fig. 9(a)]. After the multifiber probe was implanted above the MECII-
DG projection, we recorded $\mathrm{Ca}^{2+}$ signals when the mouse was freely exploring in the open field. The open field test was performed in a 1.2-m diameter round basin [Fig. 7(a)]. Examples of $\mathrm{Ca}^{2+}$ signals recorded from four channels during free exploration in the open field are shown in Fig. 9(b). $\mathrm{Ca}^{2+}$ transients were observed in three of the four channels; the channel without a detectable signal was inserted through the projection [fiber \#1 in Fig. 9(a)]. Asynchronized $\mathrm{Ca}^{2+}$ signals were detected in channel \#3 and shown in Fig. 9(b).

We calculated the correlation coefficient of the $\mathrm{Ca}^{2+}$ signals recorded from different channels in the anesthetized state and freely exploring state. We found that the correlation coefficient (a)

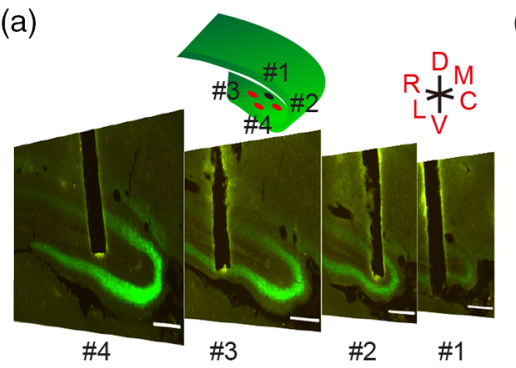

(b)

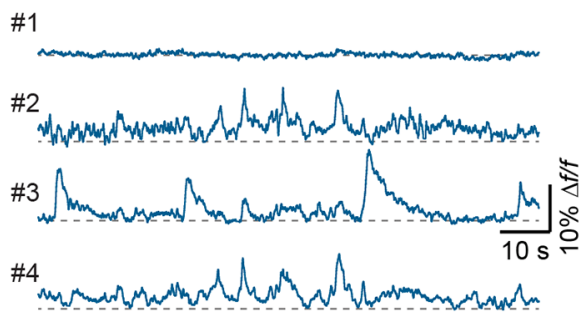

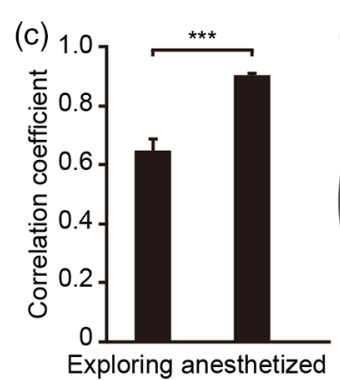

(d)

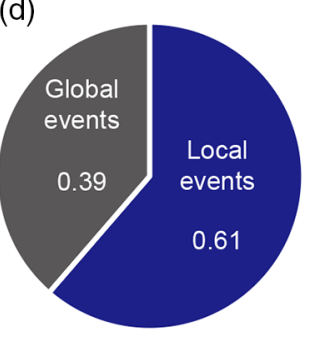

Fig. 9 Multichannel fiber recording in MECII-DG projection in freely exploring mice. (a) Post hoc histology after multichannel fiber recording showing the positions of four fibers, scale bar $200 \mu \mathrm{m}$. Inset: the relative positions of recording sites. (b) Example showing $\mathrm{Ca}^{2+}$ signals of four individual channels in MECII-DG projection during freely exploring. (c) Comparison of the correlation coefficient recorded under anesthetized and freely exploring (Wilcoxon rank-sum test, $z=6.0$, ${ }^{\star * *} p<0.001$, and $n=24$ recording sites from 6 mice). (d) Pie plot showing the fractions of local events and global events from 6 mice. R, rostral; C, caudal; D, dorsal; V, ventral; M, medial; and L, lateral. 
significantly decreased in the freely exploring state $(0.65 \pm$ $0.04)$ compared to the anesthetized state $(0.90 \pm 0.01, z=6.0$, and $p<0.001)$. These data coincided the previous results that neurons in neocortex, thalamus, and hippocampus show synchronous activity under anesthesia. ${ }^{43-45}$ We then sought out the local $\mathrm{Ca}^{2+}$ events and global $\mathrm{Ca}^{2+}$ events from four channels (see details in Sec. 2.6). Data from six mice, 24 recording sites in total, were used for analysis. The mouse with only three active channels was not used for analyses. Local $\mathrm{Ca}^{2+}$ events accounted for $61 \%$ of all the events, while the remaining events were global [Fig. 9(c)]. These results demonstrated a heterogeneous activity pattern in the subdomains of the MECII-DG projection during free exploration. These results validate the use of our multichannel fiber photometry device for functional recording in the subdomains of the MECII-DG projection.

\section{Discussion}

Two-photon $\mathrm{Ca}^{2+}$ imaging, which allows for subcellular structure resolution, is often used for projection-specific functional mapping. ${ }^{15}$ However, the mapping region is restricted to less than $200 \mu \mathrm{m} \times 200 \mu \mathrm{m} .{ }^{15,46}$ Although the recording site can be extended using a dual-axis mode, the minimum distance of the sites was restricted to $\sim 1 \mathrm{~mm}$ by the mechanical components. ${ }^{19}$ In addition, two-photon microscopy is mainly restricted to brain regions on the surface of a head-fixed animal.

Our method provides a simple way to do real-time functional monitoring in axonal terminals at multiple sites in freely behaving mice. The detector in our multichannel fiber photometry system is highly sensitive. The sCMOS camera with low noise is as sensitive as a photodetector equipped with a lock-in amplifier for recording $\mathrm{Ca}^{2+}$ signals in vivo. ${ }^{33}$ Multichannel fiber photometry using a camera as the detector, rather than a photodetector, is much simpler and easier to extend compared with other kinds of multichannel fiber photometry. ${ }^{26,32}$ Multichannel fiber photometry in the early phase is a simple mixture of single-channel fiber photometry. ${ }^{26}$ This method needs one photodetector for each channel and a more complex light splitting and coupling path. This makes it difficult to extend. Scan-based multichannel fiber photometry requires a galvanometer to scan among different channels to couple light into different channels. ${ }^{32}$ Different channel number designs require different scanning modes and data acquisition programs. However, our image-based multichannel fiber photometry is much easier to extend because the coupling of different channels is simultaneous. When we increase the number of recording channels, there is no need to make any other changes except for replacing the fiber probe.

The ability of multimode fiber to collect fluorescence increases with the increase of core diameter due to a larger NA. In general, fibers with larger core diameters are more sensitive for $\mathrm{Ca}^{2+}$ recording. But the recording range, mechanical constraint and invasiveness increases with larger core diameter fiber. Here our system used optical fibers with a core diameter of $50 \mu \mathrm{m}$. This change led to a decrease in recording depth to $<250 \mu \mathrm{m}$. However, mechanical constraint was reduced to a level that had little influence on the locomotion of the mice. Furthermore, the reduction of fiber core diameter still provided enough sensitivity and SNR to record from axonal terminals.

We also designed a fiber probe with four fibers glued in a single-metal canula. This design decreased the distance of neighboring fibers to the coating diameter of $245 \mu \mathrm{m}$. This design allowed for the recording of multiple sites within a close distance. By designing the probes according to the geometry of the recording site and creating denser fiber bundles, recording from dozens of channels is possible. The small size of the probe also saves space and lightens the weight of carrying.

Recently, researchers reported methods for multimode fiberbased imaging techniques. ${ }^{4-49}$ However, there are several limitations that prevent the application of these methods to brain tissue. Owing to randomization, thick scattering samples such as brain tissue are not suitable for this method. Furthermore, the fiber must be kept static through the whole experiment, which is impossible when measuring from freely moving animals. A fiber bundle-based imaging technique has also been used in brain imaging. ${ }^{50,51}$ However, the invasion caused by implantation was extensive and the fiber bundle imposed a significant mechanical constraint on the animal. Despite the poor spatial resolution of fiber photometry, the ability to measure structures deep in the brain in freely behaving animals has made it a popular choice in neuroscience research.

\section{Conclusion}

We designed a new version of multichannel fiber photometry that can be used for $\mathrm{Ca}^{2+}$ recording in subdomains of axonal terminals. Our method of using small-core diameter fibers reduced the recording range. It allowed the mice to freely explore the space without mechanical constraints and was sufficiently sensitive to detect $\mathrm{Ca}^{2+}$ from axonal terminals. The simultaneous light coupling strategy and compact design of the fiber probe make the system easy to extend. Finally, we validated the system by performing four-channel $\mathrm{Ca}^{2+}$ recording in the MECII-DG projection in freely moving mice. We found a heterogeneous neuronal activity pattern in the different subdomains of the MECII-DG projection during exploration. This system could have multiple applications in neuroscience research.

\section{Disclosures}

All authors declare no conflicts of interest.

\section{Acknowledgments}

This work was supported by the National Natural Science Foundation of China (Nos. 61890952, 81671106, and 31700933); "973Program” (No. 2015CB759500); the National Key Research and Development Program of China (No. 2016YFA0201403); and Science Fund for Creative Research Group of China (No. 61721092).

\section{References}

1. L. A. Gunaydin et al., "Natural neural projection dynamics underlying social behavior," Cell 157, 1535-1551 (2014).

2. J. J. Nassi and E. M. Callaway, "Parallel processing strategies of the primate visual system," Nat. Rev. Neurosci. 10, 360-372 (2009).

3. I. J. Kim et al., "Laminar restriction of retinal ganglion cell dendrites and axons: subtype-specific developmental patterns revealed with transgenic markers," J. Neurosci. 30, 1452-1462 (2010).

4. D. S. Zahm and J. S. Brog, "On the significance of subterritories in the 'accumbens' part of the rat ventral striatum," Neuroscience 50, 751-767 (1992).

5. X. Han et al., "Role of dopamine projections from ventral tegmental area to nucleus accumbens and medial prefrontal cortex in reinforcement behaviors assessed using optogenetic manipulation," Metab. Brain Dis. 32, 1491-1502 (2017).

6. E. A. Rancz et al., "High-fidelity transmission of sensory information by single cerebellar mossy fibre boutons," Nature 450, 1245-1248 (2007).

7. H. Qin et al., "A visual-cue-dependent memory circuit for place navigation," Neuron 99, 47-55.e4 (2018). 
8. W. Denk, J. H. Strickler, and W. W. Webb, "Two-photon laser scanning fluorescence microscopy," Science 248, $73-76$ (1990).

9. R. Yuste and W. Denk, "Dendritic spines as basic functional units of neuronal integration," Nature 375, 682-684 (1995).

10. J. Akerboom et al., "Optimization of a GCaMP calcium indicator for neural activity imaging," J. Neurosci. 32, 13819-13840 (2012).

11. T. W. Chen et al., "Ultrasensitive fluorescent proteins for imaging neuronal activity," Nature 499, 295-300 (2013).

12. H. Jia et al., "Dendritic organization of sensory input to cortical neurons in vivo," Nature 464, 1307-1312 (2010).

13. T. Komiyama et al., "Learning-related fine-scale specificity imaged in motor cortex circuits of behaving mice," Nature 464, 1182-1186 (2010).

14. S. Sakadzic et al., "Two-photon microscopy measurement of cerebral metabolic rate of oxygen using periarteriolar oxygen concentration gradients," Neurophotonics 3, 045005 (2016).

15. L. Petreanu et al., "Activity in motor-sensory projections reveals distributed coding in somatosensation," Nature 489, 299-303 (2012).

16. C. L. Cox et al., "Action potentials reliably invade axonal arbors of rat neocortical neurons," Proc. Natl. Acad. Sci. U. S. A. 97, 9724-9728 (2000).

17. X. Chen et al., "Functional mapping of single spines in cortical neurons in vivo," Nature 475, 501-505 (2011).

18. L. Guo et al., "Dynamic rewiring of neural circuits in the motor cortex in mouse models of Parkinson's disease," Nat. Neurosci. 18, 1299-1309 (2015).

19. J. Lecoq et al., "Visualizing mammalian brain area interactions by dual-axis two-photon calcium imaging," Nat. Neurosci. 17, 1825-1829 (2014).

20. J. N. Stirman et al., "Wide field-of-view, multi-region, two-photon imaging of neuronal activity in the mammalian brain," Nat. Biotechnol. 34, 857-862 (2016).

21. W. Zong et al., "Fast high-resolution miniature two-photon microscopy for brain imaging in freely behaving mice," Nat. Methods 14, 713-719 (2017).

22. S. L. Resendez et al., "Visualization of cortical, subcortical and deep brain neural circuit dynamics during naturalistic mammalian behavior with head-mounted microscopes and chronically implanted lenses," Nat. Protoc. 11, 566-597 (2016).

23. H. Adelsberger, O. Garaschuk, and A. Konnerth, "Cortical calcium waves in resting newborn mice," Nat. Neurosci. 8, 988-990 (2005).

24. C. Grienberger et al., "Sound-evoked network calcium transients in mouse auditory cortex in vivo," J. Physiol. 590, 899-918 (2012).

25. G. Cui et al., "Concurrent activation of striatal direct and indirect pathways during action initiation," Nature 494, 238-242 (2013).

26. H. Adelsberger et al., "Local domains of motor cortical activity revealed by fiber-optic calcium recordings in behaving nonhuman primates," Proc. Natl. Acad. Sci. U. S. A. 111, 463-468 (2014).

27. Y. Chen et al., "Sensory detection of food rapidly modulates arcuate feeding circuits," Cell 160, 829-841 (2015).

28. Q. Zhang et al., "Locomotion-related population cortical $\mathrm{Ca}^{2+}$ transients in freely behaving mice," Front. Neural Circuits 11, 24 (2017).

29. J. Yao et al., "A corticopontine circuit for initiation of urination," Nat. Neurosci. 21, 1541-1550 (2018).

30. $\mathrm{L}$. Li et al., "In vivo fiber photometry of neural activity in response to optogenetically manipulated inputs in freely moving mice," J. Innov. Opt. Health Sci. 10, 1743001 (2017).

31. K. Simone et al., "Open-source, cost-effective system for low-light in vivo fiber photometry," Neurophotonics 5, 025006 (2018).

32. Q. Guo et al., "Multi-channel fiber photometry for population neuronal activity recording," Biomed. Opt. Express 6, 3919-3931 (2015).

33. C. K. Kim et al., "Simultaneous fast measurement of circuit dynamics at multiple sites across the mammalian brain," Nat. Methods 13, 325-328 (2016).

34. J. Li et al., "Primary auditory cortex is required for anticipatory motor response," Cereb. Cortex 27, 3254-3271 (2017).

35. G. J. Broussard et al., "In vivo measurement of afferent activity with axon-specific calcium imaging," Nat. Neurosci. 21, 1272-1280 (2018).
36. A. M. Aravanis et al., "An optical neural interface: in vivo control of rodent motor cortex with integrated fiberoptic and optogenetic technology," J. Neural Eng. 4, S143-S156 (2007).

37. A. N. Yaroslavsky et al., "Optical properties of selected native and coagulated human brain tissues in vitro in the visible and near infrared spectral range," Phys. Med. Biol. 47, 2059-2073 (2002).

38. E. Stark, T. Koos, and G. Buzsaki, "Diode probes for spatiotemporal optical control of multiple neurons in freely moving animals," J. Neurophysiol. 108, 349-363 (2012).

39. G. Buzsaki and E. I. Moser, "Memory, navigation and theta rhythm in the hippocampal-entorhinal system," Nat. Neurosci. 16, 130-138 (2013).

40. M. B. Moser, D. C. Rowland, and E. I. Moser, "Place cells, grid cells, and memory," Cold Spring Harbor Perspect. Biol. 7, a021808 (2015).

41. N. M. van Strien, N. L. M. Cappaert, and M. P. Witter, "The anatomy of memory: an interactive overview of the parahippocampal-hippocampal network," Nat. Rev. Neurosci. 10, 272-282 (2009).

42. S.-J. Zhang et al., "Optogenetic dissection of entorhinal-hippocampal functional connectivity," Science 340, 1232627 (2013).

43. A. Luczak et al., "Sequential structure of neocortical spontaneous activity in vivo," Proc. Natl. Acad. Sci. U. S. A. 104, 347-352 (2007).

44. A. Stroh et al., "Making waves: initiation and propagation of corticothalamic $\mathrm{Ca}^{2+}$ waves in vivo," Neuron 77, 1136-1150 (2013).

45. M. A. Busche et al., "Rescue of long-range circuit dysfunction in Alzheimer's disease models," Nat. Neurosci. 18, 1623-1630 (2015).

46. N. J. Sofroniew et al., "A large field of view two-photon mesoscope with subcellular resolution for in vivo imaging," eLife 5, e14472 (2016).

47. Y. Choi et al., "Scanner-free and wide-field endoscopic imaging by using a single multimode optical fiber," Phys. Rev. Lett. 109, 203901 (2012).

48. T. Cizmar and K. Dholakia, "Exploiting multimode waveguides for pure fibre-based imaging," Nat. Commun. 3, 1027 (2012).

49. M. Plöschner et al., "Multimode fibre: light-sheet microscopy at the tip of a needle," Sci. Rep. 5, 18050 (2015).

50. B. A. Flusberg et al., "High-speed, miniaturized fluorescence microscopy in freely moving mice," Nat. Methods 5, 935-938 (2008).

51. P. Miao et al., "Laser speckle contrast imaging of cerebral blood flow in freely moving animals," J. Biomed. Opt. 16, 090502 (2011).

Han Qin received his undergraduate degree in optical information science and technology from Huazhong University of Science and Technology in 2013. He is a PhD candidate in optical engineering at Huazhong University of Science and Technology. His current research interests include bioinstrumentation, neurophotonics, and cognitive neuroscience.

Jian Lu received his MD in physiology from Third Military Medical University. His research interests include spatial navigation, learning, and memory.

Wenjun Jin received his $\mathrm{PhD}$ in physiology and biophysics from Peking University. His research interests include spatial navigation, learning, and memory. Major brain regions concerned are entorhinal cortex, hippocampus, and prefrontal cortex. For research, he mainly uses techniques like fiber recording, electrophysiology recording, and optogenetics.

Xiaowei Chen is a professor of physiology and neurobiology at Third Military Medical University. His group uses methods such as $\mathrm{Ca}^{2+} \mathrm{im}-$ aging, fiber recording, electrophysiology recording, and optogenetics manipulating to probe the neural circuits in cortexes and hippocampus during learning and memory.

Ling $\mathbf{F u}$ is a professor of biomedical photonics at Huazhong University of Science and Technology. Her group develops twophoton high-resolution microscopy, fiber-based microendoscopy for brain imaging, and clinical diagnosis. Her lab also builds customerdesigned $\mathrm{Ca}^{2+}$ imaging or recording systems for the neuroscience research demands. 